\title{
Influence of Different Stabilization Systems and Multiple Ultraviolet A (UVA) Aging/Recycling Steps on Physicochemical, Mechanical, Colorimetric, and Thermal-Oxidative Properties of ABS
}

\author{
Rudinei Fiorio ${ }^{1, *(1)}$, Sara Villanueva Díez ${ }^{2}$, Alberto Sánchez ${ }^{2}$, Dagmar R. D’hooge ${ }^{3,4}(\mathbb{C}$ and \\ Ludwig Cardon ${ }^{1}$ (D) \\ 1 Centre for Polymer and Material Technologies (CPMT), Department of Materials, Textiles and Chemical \\ Engineering, Ghent University, Technologiepark 130, 9052 Zwijnaarde, Belgium; ludwig.cardon@ugent.be \\ 2 Tecnalia, Sustainable Polymers, Building Technologies, Área Anardi 5, 20730 Azpeitia, \\ Gipuzkoa, Spain; sara.villanueva@tecnalia.com (S.V.D.); alberto.sanchez@tecnalia.com (A.S.) \\ 3 Centre for Textiles Science and Engineering (CTSE), Department of Materials, Textiles and Chemical \\ Engineering, Ghent University, Technologiepark 70a, 9052 Zwijnaarde, Belgium; dagmar.dhooge@ugent.be \\ 4 Laboratory for Chemical Technology (LCT), Department of Materials, Textiles and Chemical Engineering, \\ Ghent University, Technologiepark 125, 9052 Zwijnaarde, Belgium \\ * Correspondence: rudinei.fiorio@ugent.be; Tel.: +32-09-264-58-30
}

Received: 9 December 2019; Accepted: 1 January 2020; Published: 4 January 2020

\begin{abstract}
Commercially mass-polymerized acrylonitrile-butadiene-styrene (ABS) polymers, pristine or modified by stabilization systems, have been injection molded and repeatedly exposed to ultraviolet A (UVA) radiation, mechanical recycling, and extra injection molding steps to study the impact of such treatments on the physicochemical, mechanical, colorimetric, and thermal-oxidative characteristics. The work focus on mimicking the effect of solar radiation behind a window glass as relevant during the lifetime of ABS polymers incorporated in electrical and electronic equipment, and interior automotive parts by using UVA technique. The accelerated aging promotes degradation and embrittlement of the surface exposed to radiation and causes physical aging, deteriorating mechanical properties, with an expressive reduction of impact strength (unnotched: up to $900 \%$; notched: up to $250 \%$ ) and strain at break (>1000\%), as well as an increase in the yellowing index (e.g., 600\%). UV-exposition promotes a slight increase in the tensile modulus (e.g., 10\%). The addition of antioxidants (AOs) leads to a limited stabilization during the first UVA aging, although the proper $\mathrm{AO}$ formulation increases the thermal-oxidative resistance during all the cycles. Mechanical recycling promotes an increase in strain at break and unnotched impact strength alongside a slight decrease in tensile modulus, due to disruption of the brittle surface and elimination of the physical aging.
\end{abstract}

Keywords: plastic waste; circularity; accelerated aging; recycling; stabilization

\section{Introduction}

Acrylonitrile-butadiene-styrene (ABS) polymers are, among others, largely used for the manufacturing of automotive parts as well as electrical and electronic equipment [1]. As a result, ABS is one of the most common materials found in waste of end-of-life vehicles (ELV) and waste of electrical and electronic equipment (WEEE), with variations in molecular and microstructure due to different environmental experiences. For instance, many ABS products are (in)directly exposed to sunlight during their lifetime. Moreover, heat flux and mechanical stress are created in ABS-based materials during their processing, typically employing extrusion and injection molding techniques. 
It is well known that the exposition to ultravilolet (UV) radiation, heat, and stress in the presence of oxygen can promote oxidative degradation [2-5], reducing the quality and affecting the circularity of polymers. In particular, polybutadiene (PB)-containing polymers such as ABS and high-impact polystyrene (HIPS) are susceptible to both thermal- and photo-oxidation, in which the PB phase is initially degraded, reducing the elastomeric properties of the rubbery phase and inducing discoloration and losses in impact strength and toughness [6-9]. The oxidation of PB also induces degradation of the polystyrene component in the styrene-acrylonitrile (SAN) phase $[8,10]$. Notably, several additives have been used to increase the stability of polymers during their active lifetime and processing, such as antioxidants (AOs) and light stabilizers [11,12]. These additives can trap emerging free radicals generated by heat, stress, UV radiation, etc. or unstable intermediate products (e.g., hydroperoxides), transforming them into stable products, and thus reducing degradation [12].

Numerous studies already covered the effects of aging and multiple recycling steps on ABS polymer properties. For example, Bokria and Schlick [3] investigated the photodegradation of ABS exposed to ultraviolet $B(\mathrm{UVB})$ radiation and oxygen at $45^{\circ} \mathrm{C}$ and evaluated the aging effects by attenuated total reflectance Fourier transform infrared spectroscopy (ATR-FTIR). The authors concluded that degradation of ABS occurred due to the elimination of the double bonds of 1,4-butadiene, while the SAN matrix is unmodified. Major effects occurred in the exposed surface up to a depth of ca. $50 \mu \mathrm{m}$, where the butadiene structure was modified. In the unexposed surface, butadiene units were also thermally degraded. However, no degradation was observed in the center of the samples.

Pérez et al. [13] investigated the effects of reprocessing and combined recycling-accelerated aging based on UVB exposition for a wide portfolio of ABS polymer properties. The reprocessing cycles and the accelerated aging did not cause considerable modification with respect to thermal degradation, melt flow indexes, tensile moduli, and glass transition temperature $\left(T_{g}\right)$ values. However, the reprocessing promoted a reduction in the impact strength, whereas the accelerated weathering combined with the reprocessing cycles caused a substantial reduction in the tensile strength.

Boldizar and Möller [14] studied the effect of multiple cycles of extrusion and aging in air regarding the properties of conventional ABS. These authors observed a pronounced reduction in the elongation at break due to the aging, followed by an increase of this property after extrusion. The reduction in the elongation at break was associated with the physical aging (annealing) of the SAN phase as well as to the thermal-oxidative degradation of the PB phase, mainly in the surface of the material. The temperature of oxidation presented a gradual decrease with an increase in aging and recycling steps, while the aged samples presented an increase in $T_{g}$ of the SAN phase with the increase in the number of cycles.

The effect of artificial and natural weathering on the photo-degradation of ABS was also studied by Santos et al. [15]. These authors observed that degradation occurred mainly on the surface, affecting optical, mechanical, and rheological properties. Both natural and artificial aging caused a substantial decrease in the strain at break during tensile tests as a consequence of the fragile and brittle degraded surface.

Despite the progress mentioned in the previous studies, the effects of different stabilization systems on the properties of ABS polymers submitted to multiple UV accelerated aging and recycling steps are not completely known. In view of the circularity of ABS polymers, many tests need to still be performed, specifically with ultraviolet A (UVA) light which simulates the solar radiation behind a window glass - a condition commonly found during the lifetime of electrical and electronic equipment, and interior automotive parts. Therefore, the aim of the present work is to investigate (i) ABS polymer degradation with different stabilization systems; and (ii) the relevance of multiple accelerated aging and recycling steps on the physicochemical, mechanical, colorimetric, and thermo-oxidative properties. 


\section{Materials and Methods}

\subsection{Materials}

ABS Magnum 3453 Natural (Trinseo, Terneuzen, The Netherlands, obtained by mass polymerization), Irganox 1076 [octadecyl-3-(3,5-di-tert-butyl-4-hydroxyphenyl)propionate] (BASF, Ludwigshafen, Germany, phenolic primary antioxidant), Irganox 245 [ethylenebis(oxyethylene)bis-(3-(5-tert-butyl-4-hydroxy-m-tolyl)propionate)] (BASF, phenolic primary antioxidant), and Irgafos 168 [tris(2,4-ditert-butyl-phenyl)phosphite] (BASF, phosphite secondary antioxidant) were used as received unless stated otherwise.

\subsection{Methods}

\subsubsection{Extrusion-Based Manufacturing of Stabilization Masterbatches for First Injection Molding}

Two masterbatches were prepared in a micro-compounder (Minilab II, Haake Thermo Fisher Scientific, Karlsruhe, Germany) at $220{ }^{\circ} \mathrm{C}, 150 \mathrm{rpm}$, and at an extrusion rate of approximately $100 \mathrm{~g} \cdot \mathrm{h}^{-1}$. One masterbatch contained $5 \mathrm{~m} \%$ of Irganox 1076 (Masterbatch 1) and the other masterbatch contained $5 \mathrm{~m} \%$ of Irganox 1076, $5 \mathrm{~m} \%$ of Irganox 245, and $5 \mathrm{~m} \%$ of Irgafos 168 (Masterbatch 2). Before processing, the ABS (Magnum 3453 Natural) was dried at $80{ }^{\circ} \mathrm{C}$ for $4 \mathrm{~h}$ in a compressed-air dryer (CARD 40E, FarragTech, Wolfurt, Austria). Antioxidants (AOs) were used as received. After extrusion, the masterbatches were cooled by air at room temperature and granulated.

\subsubsection{Injection Molding}

Three sample types, presenting different stabilization systems, were prepared by injection molding: pristine ABS (short notation: ABS 0), ABS containing $4 \mathrm{~m} \%$ of Masterbatch 1 (short notation: ABS 1), and ABS containing $4 \mathrm{~m} \%$ of Masterbatch 2 (short notation: ABS 2). The final mass contributions of the stabilizers used in each sample type are shown in Table 1. Before processing, the ABS and the masterbatches were dried at $80^{\circ} \mathrm{C}$ for $4 \mathrm{~h}$ in a compressed-air dryer. Tensile test specimens (according to ISO 527-1A standard [16]) and bars with dimensions of $100 \mathrm{~mm} \times 10 \mathrm{~mm} \times 4 \mathrm{~mm}$ were molded in an Engel E-Victory 28T (Schwertberg, Austria) injection molding machine using a temperature profile of $250-220^{\circ} \mathrm{C}$ (nozzle-hopper), a mold temperature of $60^{\circ} \mathrm{C}$, an injection speed of $90 \mathrm{~mm} \cdot \mathrm{s}^{-1}$, an injection pressure of 820 bar, and a holding pressure of 410 bar.

Table 1. Final mass contributions of stabilizers in sample types for first injection molding step.

\begin{tabular}{|c|c|c|c|c|}
\hline Sample Type & Masterbatch $^{a}$ & Irganox $1076(\mathrm{~m} \%)$ & Irganox $245(\mathrm{~m} \%)$ & Irgafos $168(\mathrm{~m} \%)$ \\
\hline ABS 0 & - & - & - & - \\
\hline ABS 1 & 1 & 0.2 & - & - \\
\hline ABS 2 & 2 & 0.2 & 0.2 & 0.2 \\
\hline
\end{tabular}

\subsubsection{Accelerated Aging}

The accelerated aging was conducted by exposing the samples to ultraviolet A (UVA) radiation (QUV/basic, Q-Panel Lab Products, Westlake, OH, USA) during $360 \mathrm{~h}$ at $50{ }^{\circ} \mathrm{C}$ using a UVA-351 (type 1B) fluorescent UV lamp, which has a peak emission of $\lambda=353 \mathrm{~nm}$. Such conditions are used for simulation of the UV portion of solar radiation behind window glass. This experiment was conducted according to the ISO 4982 standard [17].

\subsubsection{Mechanical Recycling}

After the accelerated aging, the samples were shredded (RSP 15/30, Piovan, Santa Maria di Sala, Italy), dried ( $80^{\circ} \mathrm{C}, 4 \mathrm{~h}$ ), and extruded (Plasti-Corder, Brabender, Duisburg, Germany). The extruder 
characteristics are screw diameter $19 \mathrm{~mm}$, length/diameter ratio 25, barrel temperature profile 220-180 ${ }^{\circ} \mathrm{C}$ (die-hopper), and screw speed $60 \mathrm{rpm}$. The extruded materials were cooled in a water bath at room temperature and granulated. After recycling, the samples were dried and injection molded as previously described, followed by another accelerated aging and mechanical recycling step. Figure 1 presents the research process flow chart, covering two repetitions as highlighted with the large grey arrow in the right part of this figure.

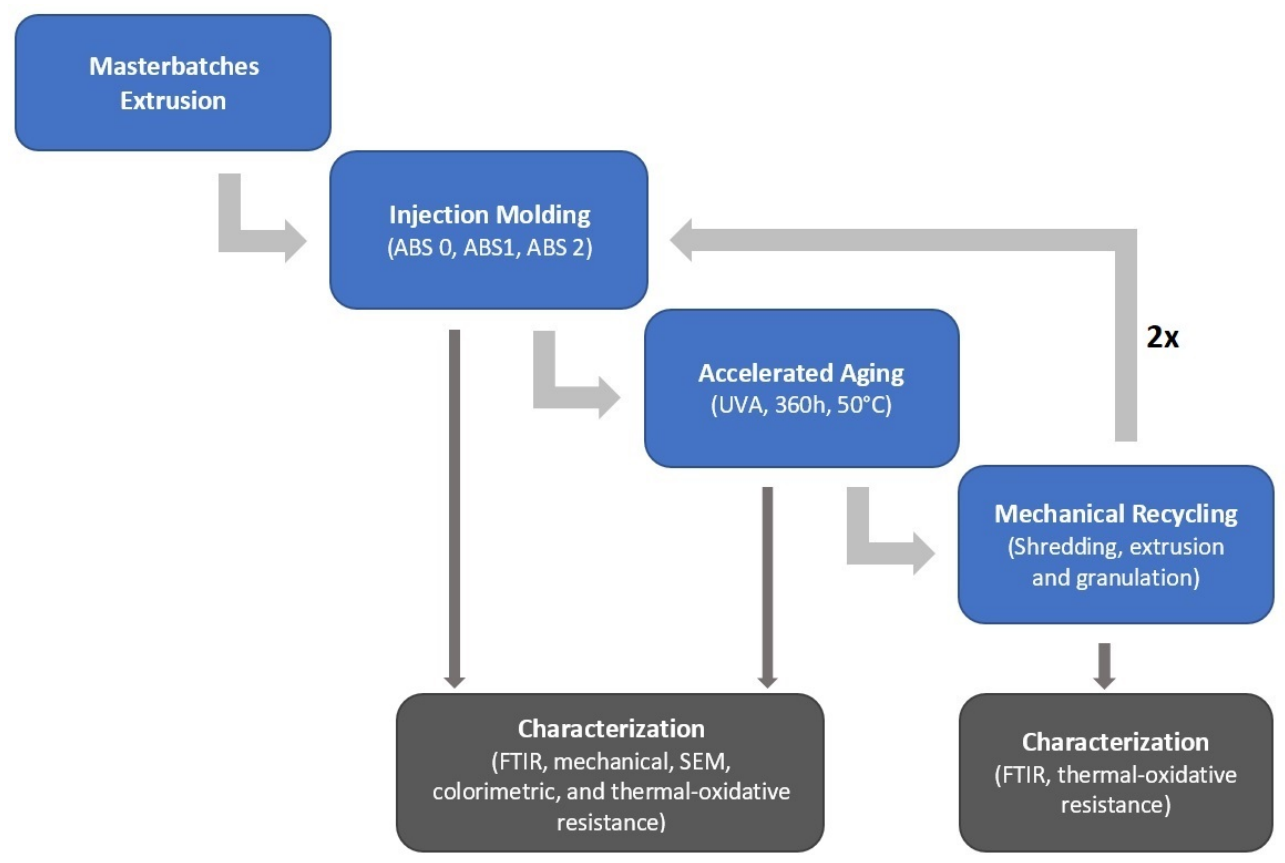

Figure 1. Research process flow chart to study the impact of various stabilization systems as combined with commercial ABS polymer (Table 1) after several accelerated aging and mechanical recycling steps.

\subsection{Characterization}

\subsubsection{Chemical Characterization}

Fourier transform infrared spectroscopy (FTIR; Bruker Tensor 27, Billerica, MA, USA) analyses were carried out in order to evaluate chemical modifications in the ABS polymer. Samples were studied in attenuated total reflectance (ATR) mode from 4000 to $600 \mathrm{~cm}^{-1}$. The values of absorbance were determined using the baseline method. An overview of relevant absorbances is provided in Table 2 . All the absorbance results were normalized considering the maximum absorbance observed for the nitrile group $(\mathrm{CN})$ at $2237 \mathrm{~cm}^{-1}$. This absorption band was chosen since the $\mathrm{CN}$ group is expected to be stable after aging and recycling steps [3,18-20].

Table 2. Overview of relevant absorbances for the FTIR spectra.

\begin{tabular}{cc}
\hline Absorbance $\left(\mathbf{c m}^{-\mathbf{1}}\right)$ & Group \\
\hline 2237 & Nitrile (CN) \\
1730 & Carbonyl (CO) \\
966 & 1,4-Butadiene (1,4BD) \\
911 & 1,2-Butadiene (1,2BD) \\
\hline
\end{tabular}

Both the unexposed and exposed to UV radiation sides of the aged samples were analyzed. To evaluate the depth of the chemical modification caused by UV aging, the surfaces of both sides of the samples were microtomed (Leica RM2245 microtome, Wetzlar, Germany) and the chemical 
structure in different depths was investigated (depth $=0,100,200$, and $300 \mu \mathrm{m})$. The carbonyl (CO, ca. $\left.1730 \mathrm{~cm}^{-1}\right)$, the 1,4-butadiene $\left(1,4 \mathrm{BD}, 966 \mathrm{~cm}^{-1}\right)$, and the nitrile $\left(\mathrm{CN}, 2237 \mathrm{~cm}^{-1}\right)$ absorption peaks were evaluated using the absorption ratios $\mathrm{CO} / \mathrm{CN}$ and $1,4 \mathrm{BD} / \mathrm{CN}$ as defined by the Equations (1) and (2), respectively [20].

$$
\begin{array}{r}
\mathrm{CO} / \mathrm{CN}=\frac{\text { Absorbance at } 1730 \mathrm{~cm}^{-1}}{\text { Absorbance at } 2237 \mathrm{~cm}^{-1}} \\
1,4 \mathrm{BD} / \mathrm{CN}=\frac{\text { Absorbance at } 966 \mathrm{~cm}^{-1}}{\text { Absorbance at } 2237 \mathrm{~cm}^{-1}}
\end{array}
$$

\subsubsection{Mechanical Properties}

The impact strength (notched and unnotched) and the tensile properties of the injection molded and UV-aged samples were investigated according to the ISO 180 and ISO 527 standards, respectively $[16,21]$.

\subsubsection{Morphological Characterization}

The morphology of the aged injection molded samples was investigated by scanning electron microscopy (SEM) in a Phenom G1 microscope (FEI Company, Hillsboro, OR, USA), using an electron acceleration voltage of $5 \mathrm{kV}$. SEM micrographs were obtained from the surfaces exposed and unexposed to UVA. The SEM samples were obtained by cutting the aged parts at room temperature, using a cutting plier. The samples were dried in an oven at $80^{\circ} \mathrm{C}$ for $4 \mathrm{~h}$ and maintained in a desiccator until the analysis.

\subsubsection{Colorimetric Characterization}

The color properties of the samples were investigated with an XPM spectrophotometer (Konica Minolta, Tokyo, Japan) using a D65 light source and $10^{\circ}$ viewing angle. All color measurements were obtained from the injection molded specimens. For the samples exposed to UV radiation, the color characteristics of both sides of the samples (exposed and unexposed sides) were analyzed. The yellowness index (YI) was used for evaluating the color modifications, as obtainable from the values of $L^{*}$ and $b^{*}$ (CIELAB color space) according to Equation (3) [22].

$$
Y I=\frac{142 \cdot 86 \cdot b^{*}}{L^{*}}
$$

\subsubsection{Thermal-Oxidative Resistance}

The thermal-oxidative resistance of all the samples was evaluated by determining the oxidation onset temperature (OOT) and the oxidation peak temperature (OPT). OOT and OPT indicate a relative degree of oxidative stability of a certain material [20]. A simultaneous thermal analyzer (STA 449 F3 Jupiter, Netzsch, Selb, Germany) was used, and the method was based on the ASTM E2009 standard [23]. For the aged samples, the thermal-oxidative resistance of both sides (exposed and unexposed to UV) were analyzed. Films of $\sim 100 \mu \mathrm{m}$ were obtained from the surfaces of the samples using a microtome. The experiments were conducted under an oxygen flow of $50 \mathrm{~mL} \cdot \mathrm{min}^{-1}$ and a sample mass of $4 \pm 1.5 \mathrm{mg}$. A Pt-Rh pan was used, and the samples were studied from 50 to $500^{\circ} \mathrm{C}$ at a heating rate of $10^{\circ} \mathrm{C} \cdot \mathrm{min}^{-1}$.

\section{Results and Discussion}

\subsection{Chemical Characterization}

Figure 2 presents the FTIR spectra of the injection molded sample ABS 0 (no extra stabilizer system; entry 1 in Table 1), before aging (injection molded; black line), and after the 1st aging step with 
surfaces exposed (red line) and unexposed to UV (blue line). After UV aging, the chemical modification of the surfaces is noticeable. Figure 2 shows an increase in the absorption peaks at $1730 \mathrm{~cm}^{-1}$ (carbonyl, $\mathrm{CO}$ ) and at 1600,1580,1070, and $1028 \mathrm{~cm}^{-1}$, indicating an oxidation of the sample, whereas the absorption peaks related to butadiene at 966 (1,4-butadiene) and $911 \mathrm{~cm}^{-1}(1,2$-butadiene) decreased with the aging, representing degradation of the rubbery phase. The degradation of the side unexposed to UV can be related to thermal aging, since the accelerated aging cycle was conducted at a temperature of $50{ }^{\circ} \mathrm{C}$ during $360 \mathrm{~h}$. Similar results were observed in previous studies involving degradation of ABS $[3,7,9,18,24-27]$. However, some authors also identified an absorption band associated to hydroxyl groups $\left(\mathrm{OH}, \mathrm{ca} .3450 \mathrm{~cm}^{-1}\right)$, which was not observed in the results of the present study. The $\mathrm{OH}$ absorption band was associated to alcohols and carboxylic acids $[3,9,18,24,26]$, with alcohols resulting from the reaction of peroxy radicals with the polymer chains [18]. The observed discrepancy between the results of the present work and the previous ones may be related to the differences among the ABS materials studied, such as the PB content and the stabilizers used.

For completeness, note that all the samples showed similar results as in Figure 2, explaining why the focus was restricted to a limited number of experiments. For the additional spectra, the reader is referred to the Supplementary Materials (Figures S1-S3).

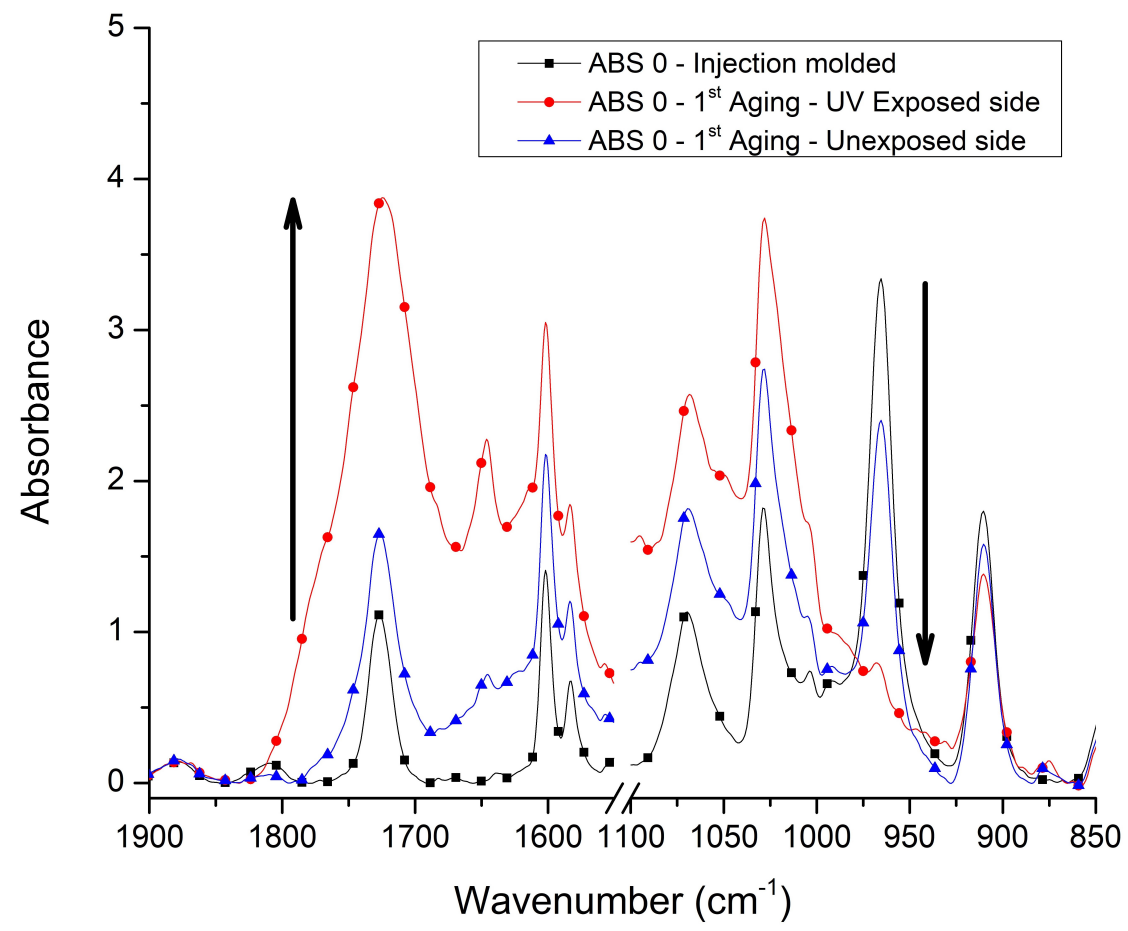

Figure 2. FTIR spectra of the sample ABS 0 (no extra stabilizer system; entry 1 in Table 1): injection molded (unaged) and after the 1st aging step (surfaces exposed and unexposed to UV). For other samples, see Supplementary Materials-Figures S1-S3.

From Figure 2 (focus on ABS 0 and only the 1st aging step), the most evident modifications caused by UV exposition were observed in the carbonyl (CO) and 1,4-butadiene (1,4BD) absorption bands. Therefore, the $\mathrm{CO} / \mathrm{CN}$ and $1,4 \mathrm{BD} / \mathrm{CN}$ absorption ratios (Equations (1) and (2), respectively) were further investigated. These absorption ratios, obtained at different depths of the side exposed to UV radiation, are presented in Figure 3 covering results for all samples and various cycles. One can clearly notice that the exposition to UVA radiation increased the $\mathrm{CO} / \mathrm{CN}$ and decreased the $1,4 \mathrm{BD} / \mathrm{CN}$ absorption ratios at the surface (depth $=0 \mu \mathrm{m})$, whereas for larger depths $(\geq 100 \mu \mathrm{m})$ these absorption ratios do not show a considerable modification compared to the values obtained for the unaged samples. 
Moreover, the change in the stabilization system, as well as the increase in the number of aging cycles, do not promote an appreciable change in the absorption ratios. In this respect, the higher $\mathrm{CO} / \mathrm{CN}$ absorption ratio observed for the sample ABS 1-3rd UV aging was considered as insignificant due to the high standard error found for this sample. Therefore, the degradation promoted by the UVA radiation was superficial $(<100 \mu \mathrm{m}$ in depth). This superficial degradation was also previously observed [3,14,15], and it is associated to the limited oxygen diffusion into the ABS and to a decrease of the absorbed light intensity through the polymer $[8,9,18]$. Changing the color characteristics due to aging could also modify the absorbance of the light and affect the degradation [18]. However, the modification of the color properties with the consecutive UV aging and recycling steps, as discussed below, does not alter the FTIR results observed in this work.
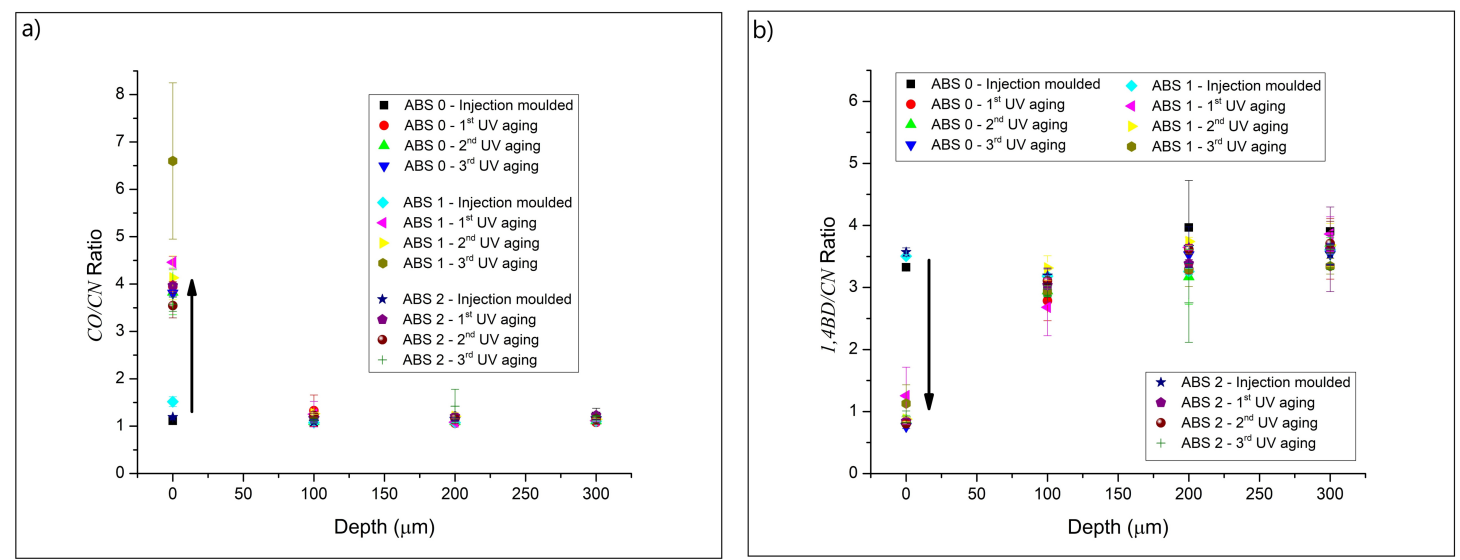

Figure 3. Absorption ratios for the side exposed to UV at different depths (Depth $0=$ surface of the samples) for samples in Table 1 (all three entries) and up to two extra cycles; (a) CO/CN ratio, and (b) 1,4BD/CN ratio (Equations (1) and (2)). Substantial degradation is observed only at the surface.

\subsection{Mechanical Properties}

\subsubsection{Impact Strength}

Figure 4 shows the results for the notched (Figure 4a; lower starting values) and unnotched (Figure $4 \mathrm{~b}$; higher starting values) impact resistance, respectively, covering the three sample types described in Table 1 and several cycles of recycling and aging, as displayed in Figure 1. One can observe in Figure $4 \mathrm{a}$ that the addition of AOs does not change the notched impact strength. However, in Figure $4 \mathrm{~b}$ a higher unnotched impact resistance is observed for the sample types containing AOs (ABS 1 and ABS 2 in Table 1) after the 1st UV exposition, showing a stabilization effect promoted by the presence of AOs.

The exposition to UV and the subsequent mechanical recycling substantially reduces both the notched (changes up to $250 \%$ ) and unnotched (changes up to $900 \%$ ) impact strength, indicating that degradation processes occurred during both accelerated aging and mechanical recycling $[3,14,15,28,29]$. A remarkable difference is noticed comparing the notched and unnotched impact strength, between the 2nd aging and the 2nd mechanical recycling treatments, as well as between the 2nd recycling and the 3rd aging, with the notched impact strength results similar for the 2nd aging and the 2nd recycling treatment. However, the unnotched impact strength is considerably higher after the 2nd mechanical recycling, in comparison with the results found for both 2nd and 3rd accelerated aged samples.

Similar behavior was observed by Boldizar and Möller [14] for the elongation at break during tensile tests, in which this elongation increased after the extrusion of accelerated aged ABS polymers. These authors associated the changes to both reversible physical aging and thermo-oxidative degradation. Moreover, Wysgoski [25] observed that thermal treatments by oven aging can promote embrittlement of ABS even without oxidation and correlated this behavior to conformational changes 
in the SAN phase due to annealing. Another possible explanation for the unnotched impact results is the formation of a degraded, brittle surface during the UV aging, which reduces the overall impact strength of the ABS-based material. This brittle surface is not present in the mechanically recycled samples since it is disrupted and homogeneously distributed in the sample during the shredding, extrusion and injection molding steps. Therefore, mechanical recycling removes the brittle surface, which can induce crack propagation [14,30], as well as eliminates the physical aging, thus increasing the unnotched impact strength.
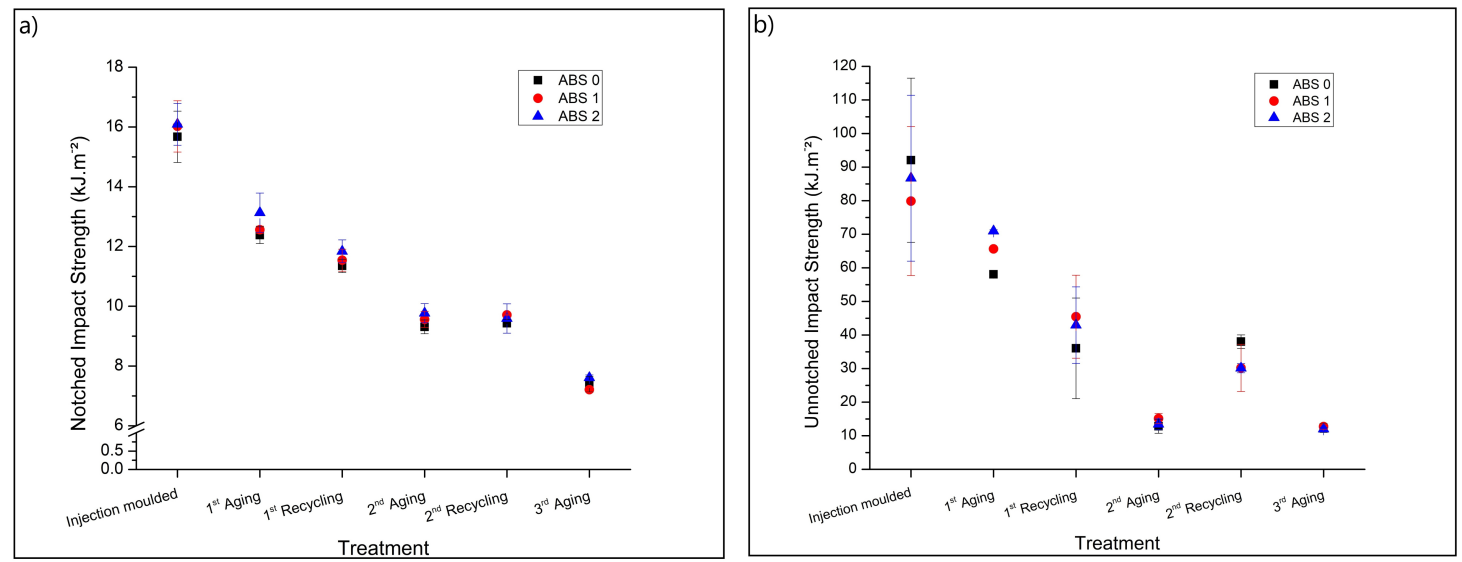

Figure 4. Impact strength of the sample types in Table 1. (a) Notched, and (b) Unnotched; testing procedure as in Figure 1.

\subsubsection{Tensile Properties}

Figure 5 shows the tensile modulus (Figure 5a) and strain at break (Figure 5b) results according to the same ordering as in Figure 4. From Figure 5a, it is observed that the 1st aging causes an increase in the tensile modulus of the sample ABS 0 (unmodified sample type), indicating modification. With the addition of AOs the UV resistance of the ABS slightly increased during the 1st aging, and the tensile modulus does not change. Both 1st and 2nd mechanical recycling step induce tensile modulus values close to those found for the injection molded (unaged) samples. The 2 nd and 3rd aging steps cause an increase in the tensile modulus for all samples. The accelerated aging promotes physical aging such as conformational changes in the SAN phase, as well as results in the formation of a brittle, degraded surface, which causes the increase in the tensile modulus values. As explained above, the recycling steps remove the effect of the physical aging, changing again the conformation of the SAN molecules [25], as well as eliminates the brittle surface, thus lowering the tensile modulus. Overall, the changes in the tensile modulus are between 5 and $10 \%$.
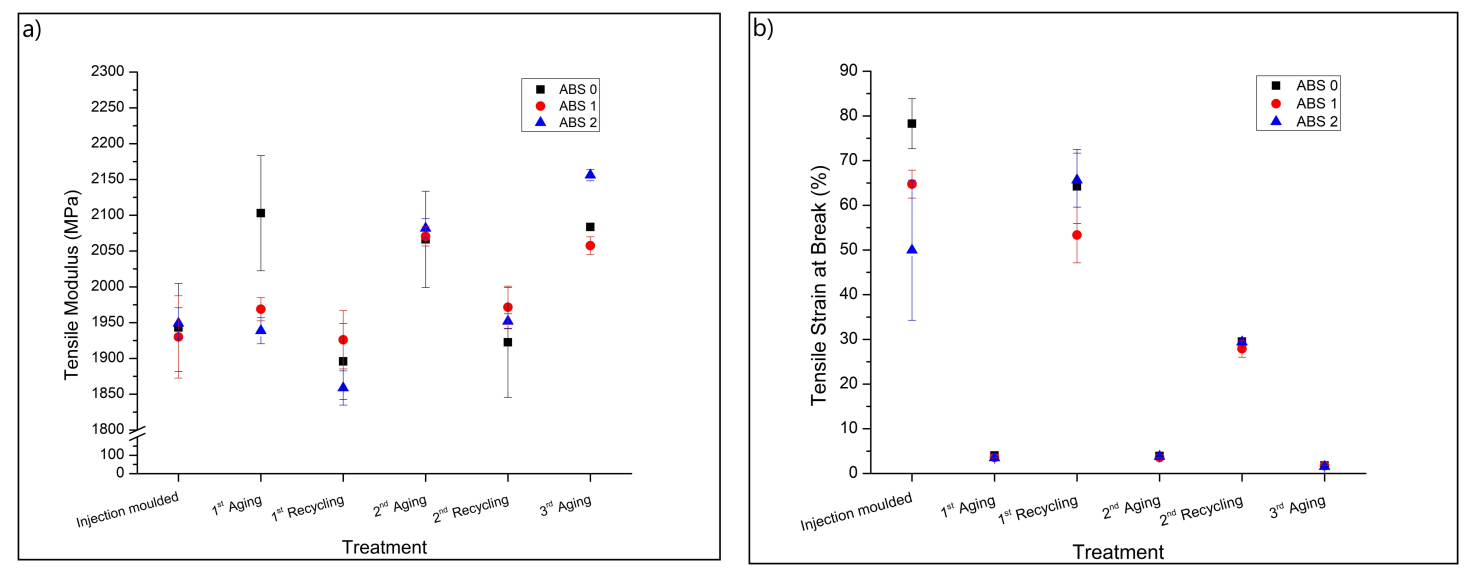

Figure 5. Tensile properties of the samples. (a) Tensile modulus, and (b) strain at break. 
Figure $5 \mathrm{~b}$ shows the strain at break for the same samples as in Figure $5 \mathrm{a}$. It is observed that the addition of AOs causes a reduction in the strain at break for the unaged (injection molded) samples, indicating that these additives can act as impurities, reducing the toughness of ABS. UV aging promotes a drastic reduction in the strain at break (factor $>10$ ), which occurs before the yield point for all UV-aged samples. The reduction in the strain at break due to accelerated aging and reprocessing of ABS was previously observed in other studies [14,15]. Once again, the dramatic reduction in the elongation at break is related to the physical aging and embrittlement of the surface. This fragile surface can induce crack propagation during the tensile deformation [15], reducing the overall toughness. The addition of AOs does not promote any noticeable modification of this property.

Compared to the UV-exposed samples, mechanical recycling leads to an increase in the strain at break since the recycling generated a more homogeneous material. However, the 2nd recycling promotes a reduction in the strain at break, compared to the result found for the 1st recycling step. Degradation occurs during the 2nd aging as well. Moreover, one can expect an increase in the concentration of degraded polymer from the aged surface, distributed in the material after the 2nd recycling, which can induce crack propagation and reduce the toughness.

Table 3 shows the tensile strength of the sample types. It is observed that the 1 st aging promotes a slight reduction of this property. However, the consecutive treatments do not promote a noticeable modification of the tensile strength. Rahimi et al. [29] also observed small changes (up to 3\%) in the tensile strength of ABS after five reprocessing cycles by injection molding. Contrarily, Pérez et al. [13] observed a substantial decrease in the tensile strength with the increase in UVB exposition time and correlated the results to crosslinking of the PB phase. The type of ABS used in this work is different than the one used by Pérez et al., and this may be the reason for the opposite results. Therefore, the tensile strength property is not able to indicate the degradation occurred due to the multiple UV aging and reprocessing steps.

Table 3. Overview of tensile strength of the sample types as covered in Table 1.

\begin{tabular}{cccc}
\hline \multirow{2}{*}{ Treatment } & \multicolumn{3}{c}{ Tensile Strength (MPa) } \\
\cline { 2 - 4 } & ABS 0 & ABS 1 & ABS 2 \\
\hline Injection molded & $32.2 \pm 0.2$ & $31.8 \pm 0.1$ & $31.9 \pm 0.1$ \\
1st Aging & $28.8 \pm 2.3$ & $27.4 \pm 1.4$ & $26.5 \pm 1.2$ \\
1st Recycling & $30.9 \pm 0.1$ & $30.6 \pm 0.1$ & $31.0 \pm 0.1$ \\
2nd Aging & $29.8 \pm 0.1$ & $28.7 \pm 0.7$ & $29.5 \pm 0.3$ \\
2nd Recycling & $30.8 \pm 0.1$ & $30.2 \pm 0.3$ & $30.4 \pm 0.5$ \\
3rd Aging & $32.0 \pm 0.1$ & $31.0 \pm 0.9$ & $30.8 \pm 0.1$ \\
\hline
\end{tabular}

\subsection{Morphological Characterization}

Figure 6 shows the scanning electron microscopy (SEM) images for the exposed (Figure 6a) and unexposed (Figure $6 \mathrm{~b}$ ) sides of ABS 2 (entry 3 in Table 1) after the 1st aging step. One can observe that the exposed surface (Figure 6a) presents micro-cracks close to the edges. These micro-cracks are not observed on the unexposed side (Figure $6 \mathrm{~b}$ ). The same behavior is observed for all the other samples (see Supplementary Materials-Figures S4-S6). The micro-cracks are formed during the cut of the samples and are observed only on the surfaces exposed to UV, as these surfaces become brittle with the degradation, as highlighted above. Iannuzzi et al. [27] observed that the surface micro-hardness increases with the aging time of ABS due to degradation, which corroborates the embrittlement observed in the present study. Besides the micro-cracks observed, no other differences between the aged and unaged surfaces are observed. 

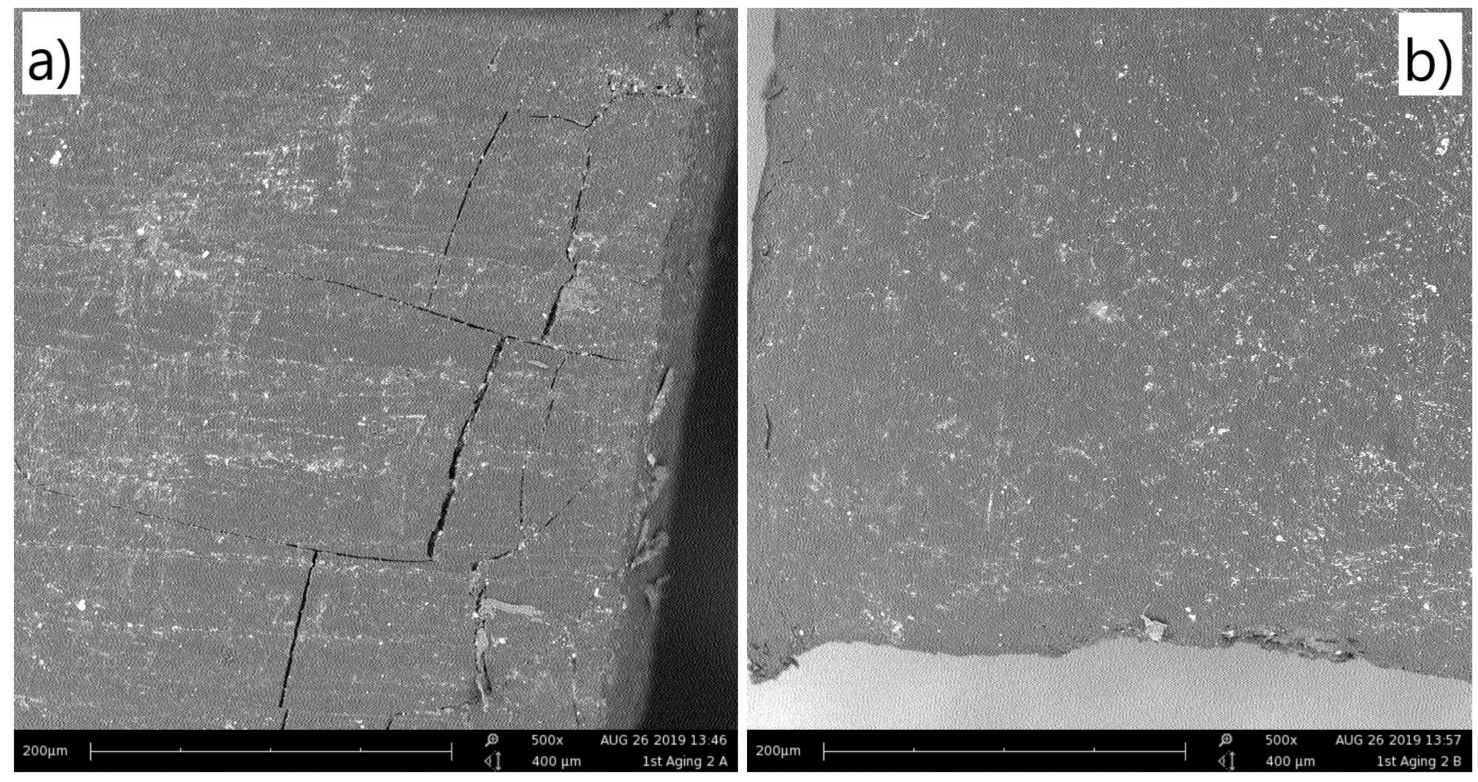

Figure 6. SEM images of the sample type ABS 2 (entry 3 in Table 1) after the 1st aging step. (a) UV exposed side and (b) unexposed side.

\subsection{Colorimetric Characterization}

Figure 7a shows the yellowing indexes (YI values) for the studied sample types from Table 1. It is observed that both accelerated aging and mechanical recycling steps cause an increase in YI, indicating that these treatments cause degradation of the polymer. Photo-oxidation of ABS increases the UV and visible light absorption, increasing the yellowing of the material [18]. The addition of AOs does not promote any noticeable stabilization of the color characteristics. The 1st recycling step promotes a large increase in the YI, possibly related to the disruption of the oxidized surface during shredding, extrusion and injection molding, which increases the surface area of the degraded superficial layer and contributes to an increase in YI.

The extrusion and injection molding of ABS induces oxidation and modifies the color characteristics, as observed by Karahaliou and Tarantili [26]. After the 2nd aging, the YI values are similar to those found for the 1st recycling step, though the 2nd recycling promotes a further increase in YI. However, the 3rd accelerated aging causes a reduction in the YI values, compared to those found after the 2 nd recycling. This reduction may be related to the formation of new chromophore groups with different color characteristics, which can cause fading [9]. Furthermore, UV aging can reduce the surface roughness of polymers [31] or induce blistering formation [9], which can also lead to color modifications.

As shown in Figure $7 \mathrm{~b}$, for the unexposed side, a slight increase in YI is observed after the 1st aging. This increase in YI values is likely related to oxidation promoted by the thermal aging (annealing at $50{ }^{\circ} \mathrm{C}$ ). This oxidation of the unexposed side is also observed in the FTIR results. Note that a sole focus on YI values is thus not recommended as each macroscopic property has a different sensitivity toward molecular and microstructural variations. 

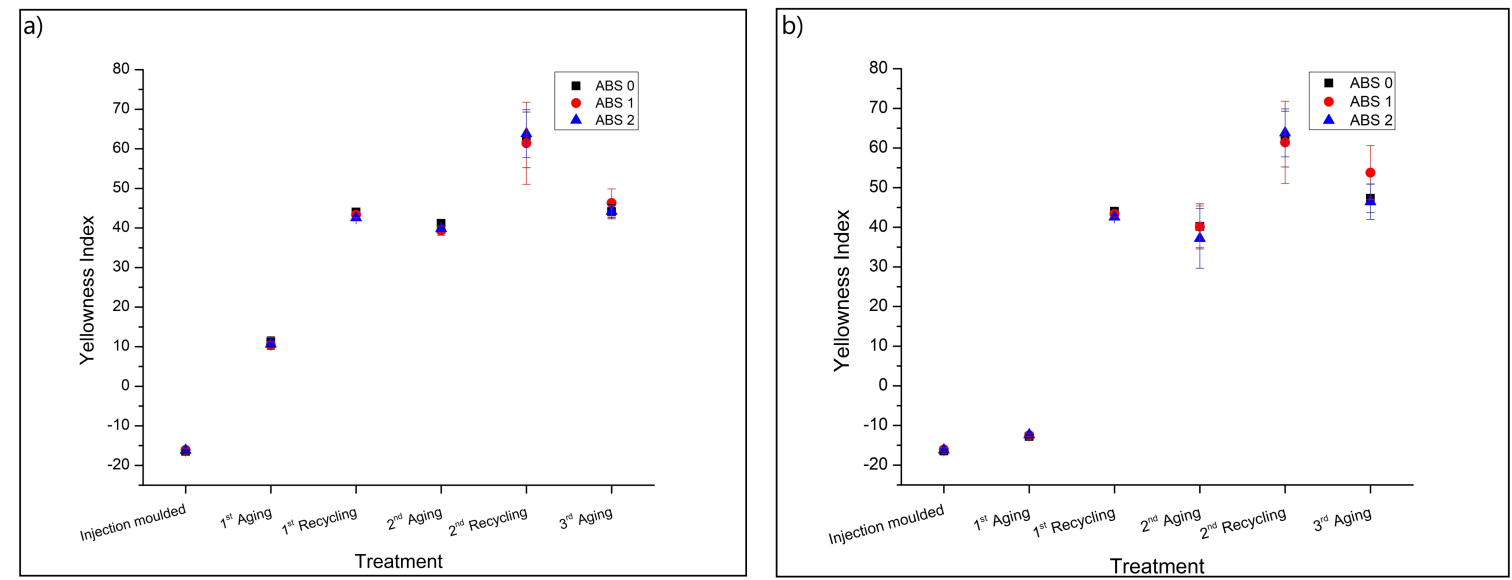

Figure 7. Yellowing index (YI) measurements. (a) UV-exposed side and (b) unexposed side for the sample types covered in Table 1, following the procedure in Figure 1.

\subsection{Thermal-Oxidative Resistance}

Figure 8 shows the corresponding oxidation onset temperature (OOT- Figure $8 \mathrm{a}$ ) and oxidation peak temperature (OPT-Figure $8 \mathrm{~b}$ ) data, including data for the sides exposed and unexposed to UV. The OOT values are obtained in the beginning of the oxidative process, while the OPT is associated to the maximum oxidation rate [20]. The higher the OOT and OPT values, the more stable the polymer is to thermal-oxidative degradation. The heat flux curves used for the determination of OOT and OPT are shown in the Supplementary Materials-Figure S7. From Figure 8, one can see that the injection molding of ABS 0 promotes a slight decrease in both OOT and OPT compared to the results found for the unprocessed (virgin) ABS (first extra data point). This expected decrease in the thermal-oxidative resistance is related to the consumption of AOs added by the polymer manufacturer and degradation occurring during processing $[12,20]$. The addition of AOs increases the thermal-oxidative resistance of ABS, as previously observed [20]. ABS 1 presents slightly higher OOT and OPT values compared to those found for ABS 0 , whereas ABS 2 showed a remarkably higher thermal-oxidative resistance, highlighting the relevance of the selection of the most appropriate mixture of stabilizers.
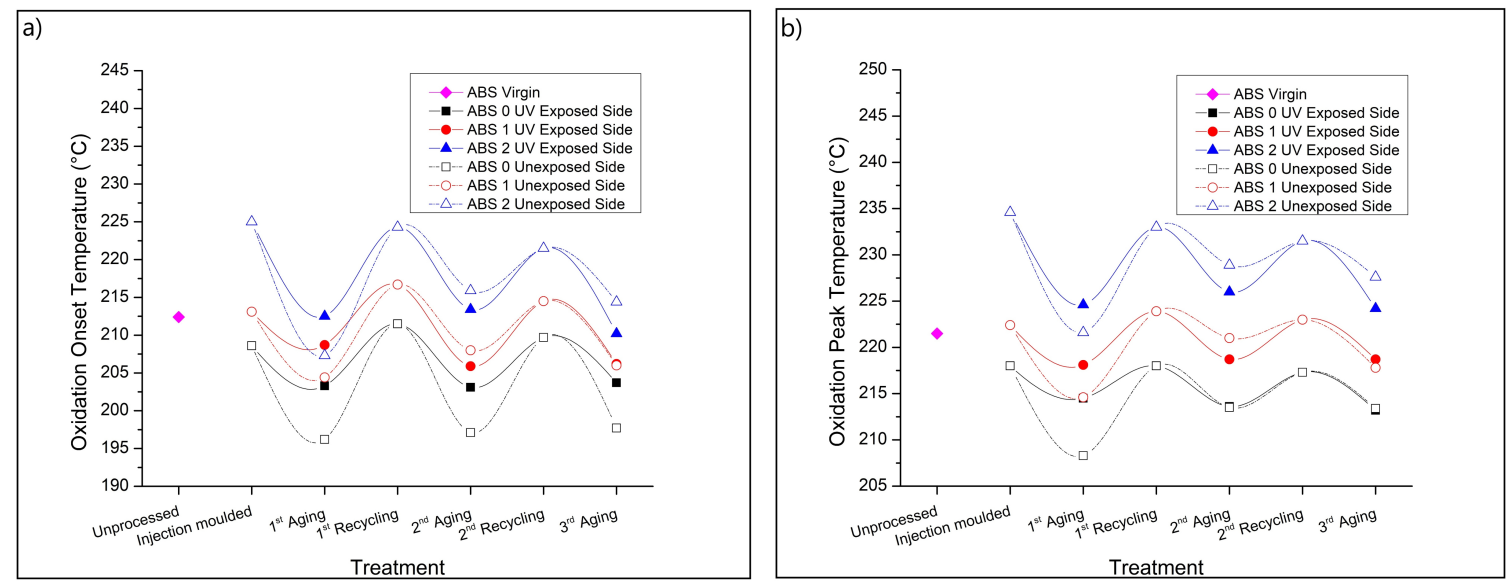

Figure 8. Thermal-oxidative resistance. (a) Oxidation onset temperatures and (b) oxidation peak temperatures for the samples covered in Table 1, following the procedure in Figure 1. Also added is the virgin ABS data point.

The effect of the sequential aging and recycling treatments on the OOT and OPT are also shown in Figure 8, with lines added for visual guidance. The smallest thermal-oxidative stability is observed for ABS 0, while the highest one is found for ABS 2. However, one can clearly observe that the accelerated 
aging promotes a reduction in the thermal-oxidative stability for both exposed and unexposed sides, while a subsequent mechanical recycling step increases the oxidative stability. Furthermore, it is observed that the stabilization systems used in the samples ABS 1 and ABS 2 are effective during all treatments, lasting up to the 3rd aging step.

It is also observed that after the 1st UV aging, the exposed sides of the samples present higher OOT and OPT values than those found for the unexposed sides. This higher thermal-oxidative stability of the exposed sides is likely related to the higher level of degradation detected for these surfaces; see the FTIR and YI results (Figures 2 and 7, respectively). These highly oxidized surfaces may present a lower oxygen diffusion rate, which should induce a higher oxidative resistance, increasing both OOT and OPT. Moreover, the reduction in the thermal-oxidative resistance of the unexposed sides are probably associated to the consumption of AOs during the aging. It is expected that aged surfaces present a lower concentration of AOs compared to the core of the sample since the AOs near the surfaces are consumed during accelerated aging [12]. The mechanical recycling promotes an increase in the homogeneity of the samples, removing the degraded surfaces and homogeneously distributing the remaining AOs in the polymer, which increases both OOT and OPT.

Figure 8 also shows that the differences between the exposed and unexposed sides for both OOT and OPT values become smaller after the 2nd and 3rd aging, compared to the results found for the 1st aging treatment. These results may be associated with an increase of the thermal-oxidative resistance of the unexposed side after the 1st aging and 1st recycling steps due to the formation of more stable compounds after the treatments, or due the formation of products that can show a stabilizing effect, as suggested by Camacho and Karlsson [32].

Boldizar et al. [14] observed a gradual decrease in the temperature of oxidation with the increase in oven-aging and recycling steps associated with the consumption of stabilizers. The differences between the results found in the present study and the previous one may be related to the different aging conditions (UVA vs. oven), as well as different types of ABS and stabilization systems.

\section{Conclusions}

In the present study, the effect of stabilization systems and consecutive UVA aging followed by mechanical recycling and injection molding on the properties of commercial ABS is investigated.

The UVA aging promotes a severe degradation of the irradiated surface $(<100 \mu \mathrm{m})$, as well as induced to physical aging (annealing). Both the embrittlement of the surfaces and the physical aging are responsible for the deterioration of the mechanical properties of the ABS. However, the core of the material, the major fraction of the polymer, does not present noteworthy degradation.

Accelerated aging and recycling steps substantially reduce the toughness of ABS. Particularly, the presence of a notch drastically reduces the impact strength, showing the importance of the design on the toughness of recycled plastic parts.

The mechanical recycling of the samples, conducted after the accelerated aging, leads to a considerable increase in the strain at break and unnotched impact strength, in addition to a slight decrease in the tensile modulus, due to disruption of the brittle surface and elimination of the thermal aging. Therefore, recycled ABS can be used for the manufacturing of new plastic parts in which high impact strength, toughness, and clear colors are not a stringent requirement.

The addition of the appropriate mixture of stabilizers increases the thermal-oxidative resistance of ABS for several aging and mechanical recycling steps, showing the endurance of the AOs. Therefore, as the correct stabilization system is added before the manufacture of ABS plastic parts, the thermal-oxidative resistance can remain adequate during service life and various recycling steps, contributing to the maintenance of the properties of the polymer and to the circular economy. 
Supplementary Materials: The following are available online at http:/ / www.mdpi.com/1996-1944/13/1/212/s1, Figure S1: FTIR spectra of the sample type ABS 0. (a) Injection molded; (b) and (c) 1st aging, UV exposed side and unexposed side, respectively; (d) and (e) 2nd aging, UV exposed side and unexposed side, respectively; (f) and (g) 3rd aging, UV exposed side and unexposed side, respectively. ' $r$ ' means repeated analysis; Figure S2: FTIR spectra of the sample type ABS 1. (a) Injection molded; (b) and (c) 1st aging, UV exposed side and unexposed side, respectively; (d) and (e) 2nd aging, UV exposed side and unexposed side, respectively; (f) and (g) 3rd aging, UV exposed side and unexposed side, respectively. ' $r$ ' means repeated analysis; Figure S3: FTIR spectra of the sample type ABS 2. (a) Injection molded; (b) and (c) 1st aging, UV exposed side and unexposed side, respectively; (d) and (e) 2nd aging, UV exposed side and unexposed side, respectively; (f) and (g) 3rd aging, UV exposed side and unexposed side, respectively. ' $r$ ' means repeated analysis; Figure S4: SEM images of the sample type ABS 0. (a) Injection molded (unaged); (b) and (c) 1st aging, UV exposed side and unexposed side, respectively; (d) and (e) 2nd aging, UV exposed side and unexposed side, respectively; (f) and (g) 3rd aging, UV exposed side and unexposed side, respectively; Figure S5: SEM images of the sample type ABS 1. (a) Injection molded (unaged); (b) and (c) 1st aging, UV exposed side and unexposed side, respectively; (d) and (e) 2nd aging, UV exposed side and unexposed side, respectively; (f) and (g) 3rd aging, UV exposed side and unexposed side, respectively; Figure S6: SEM images of the sample type ABS 2. (a) Injection molded (unaged); (b) and (c) 1st aging, UV exposed side and unexposed side, respectively; (d) and (e) 2nd aging, UV exposed side and unexposed side, respectively; (f) and (g) 3rd aging, UV exposed side and unexposed side, respectively; Figure S7: Thermal oxidative resistance of the samples. (a) Heat flux curves of the sample type ABS 0; (b) Heat flux curves of the sample type ABS 1; (c) Heat flux curves of the sample type ABS 2.

Author Contributions: Conceptualization, R.F., S.V.D., and A.S.; methodology, R.F. and S.V.D.; validation, R.F., S.V.D. and A.S.; formal analysis, R.F. and S.V.D.; investigation, R.F. and S.V.D.; resources, R.F., S.V.D., A.S., and L.C.; data curation, R.F. and S.V.D.; writing — original draft preparation, R.F.; writing-review and editing, R.F., S.V.D., A.S., D.R.D., and L.C.; visualization, R.F. and D.R.D.; supervision, L.C.; project administration, L.C.; funding acquisition, A.S. and L.C. All authors have read and agreed to the published version of the manuscript.

Funding: This research was funded by the European Union's Horizon 2020 Research and Innovation Program, grant number 730308 .

Conflicts of Interest: The authors declare no conflicts of interest.

\section{References}

1. Scaffaro, R.; Botta, L.; Di Benedetto, G. Physical properties of virgin-recycled ABS blends: Effect of post-consumer content and of reprocessing cycles. Eur. Polym. J. 2012, 48, 637-648. [CrossRef]

2. Ragaert, K.; Delva, L.; Van Geem, K. Mechanical and chemical recycling of solid plastic waste. Waste Manag. 2017, 69, 24-58. [CrossRef] [PubMed]

3. Bokria, J.G.; Schlick, S. Spatial effects in the photodegradation of poly(acrylonitrile-butadiene-styrene): A study by ATR-FTIR. Polymer 2002, 43, 3239-3246. [CrossRef]

4. Tracy, J.; D’hooge, D.R.; Bosco, N.; Delgado, C.; Dauskardt, R. Evaluating and predicting molecular mechanisms of adhesive degradation during field and accelerated aging of photovoltaic modules. Prog. Photovolt. Res. Appl. 2018, 26, 981-993. [CrossRef]

5. Yu, L.; Yan, X.; Fortin, G. Effects of weathering aging on mechanical and thermal properties of injection molded glass fiber reinforced polypropylene composites. J. Polym. Res. 2018, 25, 247. [CrossRef]

6. Scott, G.; Tahan, M. Comparison of the photo-oxidative behaviour of some polybutadiene-based polyblends. Eur. Polym. J. 1977, 13, 981-987. [CrossRef]

7. Adeniyi, J. Clarification and discussion of chemical transformations involved in thermal and photo-oxidative degradation of ABS. Eur. Polym. J. 1984, 20, 291-299. [CrossRef]

8. Jouan, X.; Gardette, J. Photo-oxidation of ABS: Part 2-Origin of the photodiscoloration on irradiation at long wavelengths. Polym. Degrad. Stab. 1992, 36, 91-96. [CrossRef]

9. Davis, P.; Tiganis, B.; Burn, L. The effect of photo-oxidative degradation on fracture in ABS pipe resins. Polym. Degrad. Stab. 2004, 84, 233-242. [CrossRef]

10. Piton, M.; Rivaton, A. Photo-oxidation of ABS at long wavelengths ( $\lambda>300 \mathrm{~nm})$. Polym. Degrad. Stab. 1997, 55, 147-157. [CrossRef]

11. Scott, G. Atmospheric oxidation and antioxidants. In Color in Food: Technological and Psychophysical Aspects; Scott, G., Ed.; Elsevier: Amsterdam, The Netherlands, 1993; Chapter 5, pp. 279-326.

12. Zweifel, H. Stabilization of Polymeric Materials; Springer: Berlin, Germany, 1998.

13. Pérez, J.; Vilas, J.; Laza, J.; Arnáiz, S.; Mijangos, F.; Bilbao, E.; León, L. Effect of reprocessing and accelerated weathering on ABS properties. J. Polym. Environ. 2010, 18, 71-78. [CrossRef] 
14. Boldizar, A.; Möller, K. Degradation of ABS during repeated processing and accelerated ageing. Polym. Degrad. Stab. 2003, 81, 359-366. [CrossRef]

15. Santos, R.; Botelho, G.; Machado, A. Artificial and natural weathering of ABS. J. Appl. Polym. Sci. 2010, 116, 2005-2014. [CrossRef]

16. ISO-527-1. Plastics-Determination of Tensile Properties-Part 1: General Principles; Technical Report; ISO: Geneva, Switzerland, 2006.

17. ISO-4892-3. Plastics-Methods of Exposure to Laboratory Light Sources—Part 3: Fluorescent UV Lamps; Technical Report; ISO: Geneva, Switzerland, 2006.

18. Jouan, X.; Gardette, J.L. Photooxidation of ABS at long-wavelengths. J. Polym. Sci. Part A Polym. Chem. 1991, 29, 685-696. [CrossRef]

19. Celina, M.; Wise, J.; Ottesen, D.; Gillen, K.; Clough, R. Oxidation profiles of thermally aged nitrile rubber. Polym. Degrad. Stab. 1998, 60, 493-504. [CrossRef]

20. Fiorio, R.; D'hooge, D.R.; Ragaert, K.; Cardon, L. A Statistical Analysis on the Effect of Antioxidants on the Thermal-Oxidative Stability of Commercial Mass-and Emulsion-Polymerized ABS. Polymers 2019, 11, 25. [CrossRef]

21. ISO-180. Plastics-Determination of Izod Impact Strength; Technical Report; ISO: Geneva, Switzerland, 2000.

22. Hirschler, R. Whiteness, yellowness, and browning in food colorimetry. In Color in Food: Technological and Psychophysical Aspects; Caivano, J., Buera, M., Eds.; CRC Press: Boca Raton, FL, USA, 2012; Chapter 10, pp. 93-104.

23. ASTM-E2009. Standard Test Methods for Oxidation Onset Temperature of Hydrocarbons by Differential Scanning Calorimetry; Technical Report; ASTM: West Conshohocken, PA, USA, 2014.

24. Adeniyi, J.; Kolawole, E. Thermal and photo-degradation of unstabilized ABS. Eur. Polym. J. 1984, $20,43-47$. [CrossRef]

25. Wyzgoski, M.G. Effects of oven aging on ABS, poly (acrylonitrile-butadiene-styrene). Polym. Eng. Sci. 1976, 16, 265-269. [CrossRef]

26. Karahaliou, E.K.; Tarantili, P. Stability of ABS compounds subjected to repeated cycles of extrusion processing. Polym. Eng. Sci. 2009, 49, 2269-2275. [CrossRef]

27. Iannuzzi, G.; Mattsson, B.; Rigdahl, M. Color changes due to thermal ageing and artificial weathering of pigmented and textured ABS. Polym. Eng. Sci. 2013, 53, 1687-1695. [CrossRef]

28. Chiu, H.T.; Huang, J.K.; Kuo, M.T.; Huang, J.H. Characterisation of PC/ABS blend during 20 reprocessing cycles and subsequent functionality recovery by virgin additives. J. Polym. Res. 2018, 25, 124. [CrossRef]

29. Rahimi, M.; Esfahanian, M.; Moradi, M. Effect of reprocessing on shrinkage and mechanical properties of ABS and investigating the proper blend of virgin and recycled ABS in injection molding. J. Mater. Process.Technol. 2014, 214, 2359-2365. [CrossRef]

30. Carter, R., III; McCallum, J. Photoacoustic infrared spectroscopy for evaluation of an ABS as an automotive interior material. Polym. Degrad. Stab. 1994, 45, 1-10. [CrossRef]

31. Lu, T.; Solis-Ramos, E.; Yi, Y.; Kumosa, M. UV degradation model for polymers and polymer matrix composites. Polym. Degrad. Stab. 2018, 154, 203-210. [CrossRef]

32. Camacho, W.; Karlsson, S. Assessment of thermal and thermo-oxidative stability of multi-extruded recycled PP, HDPE and a blend thereof. Polym. Degrad. Stab. 2002, 78, 385-391. [CrossRef]

(C) 2020 by the authors. Licensee MDPI, Basel, Switzerland. This article is an open access article distributed under the terms and conditions of the Creative Commons Attribution (CC BY) license (http://creativecommons.org/licenses/by/4.0/). 